\title{
Modeling and Control of a Spherical Rolling Robot: A Decoupled Dynamics Approach
}

\author{
Erkan Kayacan and Zeki Y. Bayraktaroglu and Wouter Saeys
}

\begin{abstract}
This paper presents the results of a study on the dynamical modeling, analysis and control of a spherical rolling robot. The rolling mechanism consists of a $2-\mathrm{DOF}$ pendulum located inside a spherical shell with freedom to rotate about the transverse and longitudinal axis. The kinematics of the model has been investigated through the classical methods with rotation matrices. Dynamic modeling of the system is based on the Euler-Lagrange formalism. Nonholonomic and highly nonlinear equations of motion have then been decomposed into two simpler sub-systems through the decoupled dynamics approach. A feedback linearization loop with fuzzy controllers has been designed for the control of the decoupled dynamics. Rolling of the controlled mechanism over linear and curvilinear trajectories has been simulated by using the proposed decoupled dynamical model and feedback controllers. Analysis of radius of curvature over curvilinear trajectories has also been investigated.
\end{abstract}

Index Terms-Spherical rolling robot, Decoupled dynamics, Feedback linearization.

\section{INTRODUCTION}

$\mathbf{M}$ OBILE robots are widely used in a variety of nonindustrial applications such as security surveillance, search and rescue, children education, entertainment, etc. Spherical rolling mechanisms exhibit a number of advantages with respect to wheeled and legged mechanisms. All mechanical and electrical components including the actuation mechanism are safely located inside a spherical shell rolling itself over the ground surface. As the motion of a sphere rolling without slipping over a surface is governed by nonholonomic constraints, spherical rolling robots are classified as non-holonomic mechanical systems.

The fundamental difference between spherical and wheeled rolling motions is the instantaneous number of DOF between the mobile body and ground surface. Since the sphere can simultaneously rotate about the transverse and longitudinal axes, its instantaneous mobility is greater than that of a wheel. Spherical rolling mechanisms can change their direction of motion easier than wheeled mechanisms. Unlike wheeled and legged mechanisms, spheres do not fall over. The general problem of stability of equilibrium frequently encountered in mobile robotics is naturally avoided by using spherical rolling.

E. Kayacan is with BIOSYST - MeBioS, Faculty of Bioscience Engineering, KU Leuven, Kasteelpark Arenberg 30, B-3001 Leuven, Belgium e-mail: erkan.kayacan@biw.kuleuven.be

Zeki Y. Bayraktaroglu is with Department of Mechanical Engineering, Istanbul Technical University, 34437 Istanbul, Turkey e-mail: zeki.bayraktaroglu@itu.edu.tr

Wouter Saeys is with BIOSYST - MeBioS, Faculty of Bioscience Engineering,, KU Leuven, Kasteelpark Arenberg 30, B-3001 Leuven, Belgium e-mail: wouter.saeys@biw.kuleuven.be
However, the dynamics of spherical rolling systems can be described by highly complex nonlinear equations.

A number of spherical robot prototypes have been developed in recent years. One of the proposed mechanical structures is constituted by a wheeled vehicle located at the bottom of the sphere. In such a scheme, the motion is provided by the interaction of the vehicle's wheels and the sphere. The wheeled vehicle's motion inside the sphere being also non-holonomic, the overall system is the combination of two non-holonomic mechanical systems [1], [2]. Camicia proposed a linear model for the longitudinal dynamics of the vehicle to simplify the equations of motion of the system [3]. They concluded that the linearization approach is not feasible for the study of this system. A similar design utilizes a single wheel resting on the bottom of the sphere [4].

In another mechanical design, the driving motion of the rolling robot is obtained by changing the position of the mass center inside the sphere, which provides a gravitational torque [5]-[7]. For example, a spherical robot with one DOF pendulum as actuation mechanism has been designed by [8]. Joshi proposed a design where two DC motors associated with two flywheels are mounted inside the sphere [9], [10]. When one of the flywheels turns about an arbitrarily chosen reference direction, the spherical rolling robot rolls in the opposite direction, due to the conservation of angular momentum. However, such a mechanism cannot roll over curvilinear trajectories, because in order to change its direction of motion, the sphere must stop rolling and then turn left or right around the vertical axis.

Several modeling approaches have been proposed for representing the dynamics of the spherical rolling motion. Firstorder mathematical models of the spherical rolling are based on the principle of the pure rolling constraint and conservation of angular momentum [11], [12]. The dynamics of a rolling sphere on a smooth surface has been modeled by using the Lagrangian method and Euler angles [13], [14]. BoltzmannHamel equation [15] and Kane method [16] are also used in modeling of spherical rolling motion.

As can be seen from the previously published research on the subject, highly nonlinear equations constituting the dynamic model are not suitable for analytic or numerical solutions. A simpler dynamic model is required for the simulation of dynamic behavior especially over curvilinear trajectories. Simplification of the equations of motion can be achieved through the decoupled dynamics approach, which has been widely used in modeling of complex dynamic systems. For example, in the analysis of underwater vehicles, the yaw, pitch, roll and vertical motion are assumed to be decoupled 
from each other [17], [18]. Also, terrestrial wheeled vehicle dynamics has been analyzed by decoupling the lateral and yaw motions of a car in order to obtain simplified steering models [19], [20]. Dynamics and control of autonomous unicycles and bicycles [21], [22] and turbofan engine modeling are other applications where decoupled dynamics has been used as a modeling approach [23]. In wheeled robots, equations of motion are usually decoupled with respect to present control inputs driving the mechanism [24].

This paper is organized as follows. The description of the proposed system is given in Section II. Section III deals with kinematic and dynamic modeling as well as decoupling of the equations of motion. Rolling over curvilinear trajectories is also analyzed in this section. In Section IV, the proposed motion controllers are described. Simulation results and comparisons are presented in Section V. Finally a brief discussion of the study is given in Section VI.

\section{SYSTEM DESCRIPTION}

The spherical rolling mechanism considered in this study consists of a fully actuated 2-DOF pendulum rotating about its transversal and longitudinal axis. The decoupled dynamics approach consists in decoupling the dynamics of the two DOF of the actuation mechanism. In other words, it is assumed that the dynamics of one DOF does not affect the dynamics of the other one [17]. Therefore the dynamic model of two subsystems can be written independently from each other. The Euler angles are used to describe the relative angular motions of the rigid bodies constituting the entire system, i.e. the spherical shell and actuation mechanism. The equations of motion are obtained through the Euler-Lagrange method. Control of rolling motion of the proposed spherical mechanism is investigated by dynamic simulations. Feedback linearization is achieved with a computed torque scheme with fuzzy controllers.

Formulation of the kinematic and dynamic equations governing the motion of the spherical rolling mechanism with a pendulum is based on the following assumptions:

1) The sphere rolls over a perfectly horizontal surface without slipping.

2) The center of mass of the entire system is also the geometric center of the spherical shell.

3) The pendulum is in vertical downward position when the sphere is in static equilibrium.

A schematic illustration of the spherical rolling robot is given in Fig. 1. $R_{f}, R_{f_{0}}$ and $R_{f_{1}}$ denote respectivelythe reference frames $O-X Y Z, O_{0}-X_{0} Y_{0} Z_{0}$ and $O_{1}-X_{1} Y_{1} Z_{1}$. $R_{f}$ represents the inertial reference frame fixed to the motion ground. $R_{f_{0}}$ is a moving frame attached to the center of the sphere and allowed to translate only with respect to $R_{f}$. $R_{f_{1}}$ is another moving frame attached also to the center of the sphere but allowed to rotate only with respect to $R_{f_{0}}$. Relative angular positions between these frames can be describe by several methods such as Euler angles, Tait-Bryan angles, RollPitch-Yaw angles, etc...

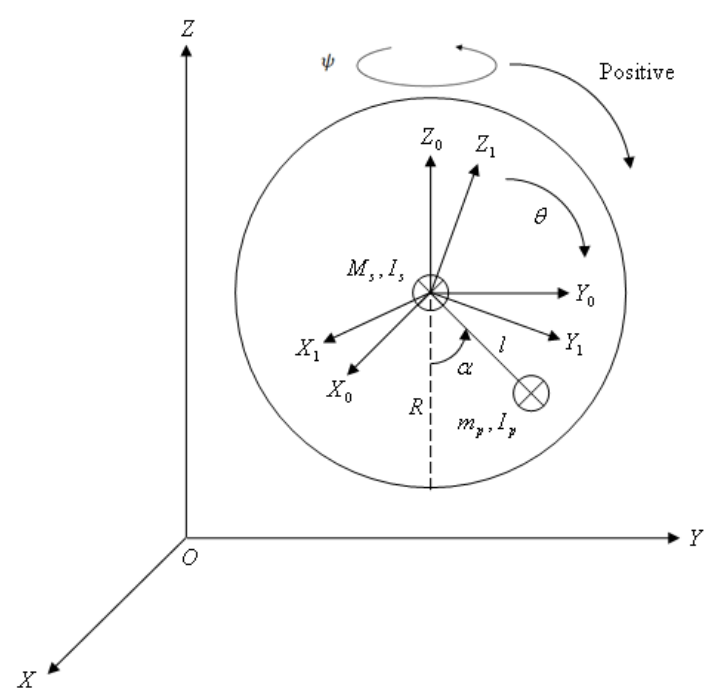

Fig. 1. Modeling of Rolling Motion About Transversal Axis For Overall Translation Along $\mathrm{O}-\mathrm{y}$

\section{A. Non-Holonomic Constraints}

The first assumption of rolling without slipping of a sphere can be mathematically described by the following constraint equations:

$$
\begin{array}{r}
F_{x}=R \omega_{y}=\dot{x}=R\left(\dot{\delta}_{1} \sin \delta_{2}-\dot{\delta}_{3} \sin \delta_{1} \cos \delta_{2}\right) \\
F_{y}=R \omega_{x}=-\dot{y}=R\left(\dot{\delta}_{1} \cos \delta_{2}+\dot{\delta}_{3} \sin \delta_{1} \sin \delta_{2}\right)
\end{array}
$$

where $\dot{x}$ and $\dot{y}$ represent the linear velocity of the center of the sphere, $\delta_{1}, \delta_{2}, \delta_{3}$ the Euler angles, $\omega_{x}$ the angular velocity of the sphere around the transversal axis, $\omega_{y}$ is the angular velocity of the sphere around the longitudinal axis [25].

For the two-dimensional motion of the sphere in the $O-x y$ plane, equations (1) constitute the so-called non-holonomic constraints which are not integrable by definition. These nonholonomic constraint equations must be handled simultaneously with the dynamical equations governing the rolling motion.

In some of the previously published related works, researchers have replaced the expressions of $\dot{x}$ and $\dot{y}$ given by the non-holonomic constraints (1) directly in the Lagrangian function of the system [26]. It has then been reported that this erroneous attempt would give correct results in some special cases like the rolling motion of a coin. However, it does not hold in general [25].

The correct equations of motion of a rolling sphere obtained through the Euler-Lagrange method are written as follows:

$$
\begin{aligned}
M^{s} \ddot{x} & =-\mu_{x} \\
M^{s} \ddot{y} & =\mu_{y} \\
I^{s}\left(\ddot{\delta}_{3}+\ddot{\delta}_{2} \cos \delta_{1}-\dot{\delta}_{2} \dot{\delta}_{1} \sin \delta_{1}\right) & =R \sin \delta_{1}\left(-\mu_{x} \cos \delta_{2}\right. \\
& \left.+\mu_{y} \sin \delta_{2}\right) \\
I^{s}\left(\ddot{\delta}_{1}+\dot{\delta}_{2} \dot{\delta}_{3} \sin \delta_{1}\right) & =R\left(\mu_{x} \sin \delta_{2}+\mu_{y} \cos \delta_{2}\right) \\
I^{s}\left(\ddot{\delta}_{2}+\ddot{\delta}_{3} \cos \delta_{1}-\dot{\delta}_{3} \dot{\delta}_{1} \sin \delta_{1}\right) & =0
\end{aligned}
$$


where $\mu_{x}$ and $\mu_{y}$ represent the Lagrange multipliers. $M^{s}$ and $I^{s}$ denote respectively the mass and moment of inertia of the sphere. The equations of motion must be handled with the non-holonomic constraints (1).

\section{Modeling of SPHERICAL Rolling Motion}

\section{A. Rolling of a Sphere with 2-DOF Pendulum}

Forward and turning motions of the rolling sphere are schematically represented in Fig. 1 and Fig. 2 respectively. System parameters are presented in Table I.

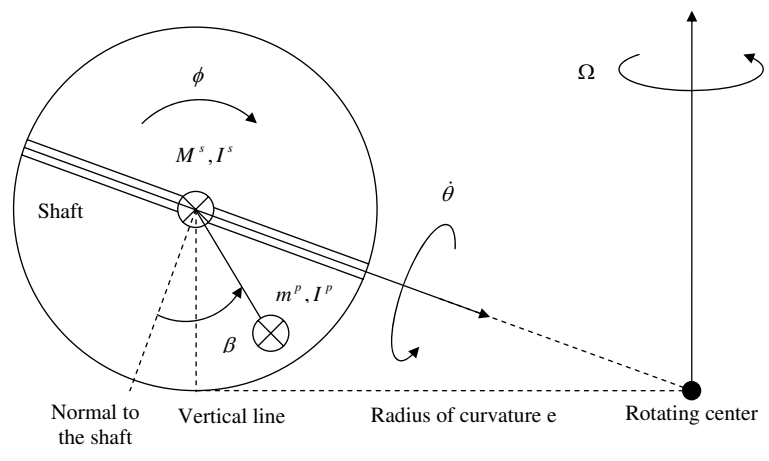

Fig. 2. Modeling of Rolling Motion About Longitudinal Axis For Overall Translation Along $O-x$

TABLE I

NOMENCLATURE

\begin{tabular}{cc}
\hline$R_{f}$ & $O-x y z$ \\
$R_{f_{0}}$ & $O-x_{0} y_{0} z_{0}$ \\
$R_{f 1}$ & $O-x_{1} y_{1} z_{1}$ \\
$O-x_{1}$ & Transversal Axis \\
$O-y_{1}$ & Longitudinal Axis \\
$\theta, \phi, \psi$ & Rolling angle of the sphere about the $\mathrm{x}, \mathrm{y}$ and $\mathrm{z}$ axes \\
$\alpha, \beta$ & Degrees of freedom of pendulum \\
$\delta_{1}, \delta_{2}$ and $\delta_{3}$ & Euler angles \\
$\mathrm{R}$ & Radius of the sphere \\
1 & Distance between the center of the sphere and \\
$\mathrm{g}$ & the center of the pendulum \\
$\Omega$ & Gravitational acceleration \\
$\omega$ & Angular rate of turning \\
$F_{c 1}$ & Angular velocity of the system during turning motion \\
$F_{c 2}$ & Centrifugal force on the sphere \\
$F_{f}$ & Centrifugal force on the pendulum \\
$N$ & Friction force \\
$\mathrm{e}$ & Reaction force \\
\hline
\end{tabular}

1) Kinematic Model: Since the sphere rotates around the transversal and longitudinal axes, the transformation matrices between $R_{f_{0}}$ and $R_{f_{1}}$ are written as follows:

$$
\begin{gathered}
T_{1 x}^{0}(\theta)=\left[\begin{array}{ccc}
1 & 0 & 0 \\
0 & \cos \theta & \sin \theta \\
0 & -\sin \theta & \cos \theta
\end{array}\right] \\
T_{1 y}^{0}(\phi)=\left[\begin{array}{ccc}
\cos (\phi) & 0 & -\sin (\phi) \\
0 & 1 & 0 \\
\sin (\phi) & 0 & \cos (\phi)
\end{array}\right]
\end{gathered}
$$

where $T_{1 x}^{0}$ and $T_{1 y}^{0}$ represent rotation around $\mathrm{x}$ and $\mathrm{y}$ axes, respectively.

The angular velocity $\omega^{\mathbf{s}}$ and linear velocity $\mathbf{v}^{\mathbf{s}}$ of the (center of the) sphere are given by:

$$
\begin{gathered}
\omega_{\mathbf{x}}^{\mathbf{s}}=-\dot{\theta} \mathbf{i} \\
\mathbf{v}_{\mathbf{x}}^{\mathbf{s}}=-R \dot{\theta} \mathbf{j} \\
\omega_{\mathbf{y}}^{\mathbf{s}}=-\dot{\phi} \mathbf{j} \\
\mathbf{v}_{\mathbf{y}}^{\mathbf{s}}=R \dot{\phi} \mathbf{i}
\end{gathered}
$$

where $\theta, \phi, \mathbf{i}$ and $\mathbf{j}$ represent rolling angle of the sphere around $\mathrm{x}, \mathrm{y}$ axes, unit vector on $\mathrm{x}$ and $\mathrm{y}$ axes, respectively.

The position vector $\mathbf{r}^{\mathbf{p}_{1}}$ of the mass center of the pendulum defined in $R_{f_{1}}$ can be written as:

$$
\begin{aligned}
\mathbf{r}_{\mathbf{x}}^{\mathbf{p}_{\mathbf{1}}} & =l \sin \alpha \mathbf{j}-l \cos \alpha \mathbf{k} \\
\mathbf{r}_{\mathbf{y}}^{\mathbf{p}_{\mathbf{1}}} & =-l \sin \beta \mathbf{i}-l \cos \beta \mathbf{k}
\end{aligned}
$$

where $\alpha, \beta$ and $\mathbf{k}$ represent rotation of the pendulum around $\mathrm{x}, \mathrm{y}$ axes and unit vector on $\mathrm{z}$ axis, respectively.

The position vector $\mathbf{r}^{\mathbf{p}_{\mathbf{o}}}$ of the mass center of the pendulum defined in $R_{f_{0}}$ can then be calculated as:

$$
\begin{aligned}
\mathbf{r}_{\mathbf{x}}^{\mathbf{p}_{\mathbf{0}}} & =T_{1 x}^{0} \mathbf{r}_{\mathbf{x}}^{\mathbf{p}_{\mathbf{1}}} \\
\mathbf{r}_{\mathbf{x}}^{\mathbf{p}_{\mathbf{0}}} & =l \sin (\alpha-\theta) \mathbf{j}-l \cos (\alpha-\theta) \mathbf{k} \\
\mathbf{r}_{\mathbf{y}}^{\mathbf{p}_{\mathbf{0}}} & =T_{1 y}^{0} \mathbf{r}_{\mathbf{y}}^{\mathbf{p}_{\mathbf{1}}} \\
\mathbf{r}_{\mathbf{y}}^{\mathbf{p}_{\mathbf{0}}} & =-l \sin (\beta-\phi) \mathbf{i}-l \cos (\beta-\phi) \mathbf{k}
\end{aligned}
$$

The position vector $\mathbf{r}^{\mathbf{p}}$ of the mass center of the pendulum defined in $R_{f}$ is identical to $\mathbf{r}^{\mathbf{p}_{\mathbf{o}}}$.

The angular velocity vectors $\omega^{\mathbf{p}_{1}}$ and $\omega^{\mathbf{p}_{\mathbf{0}}}$ of the pendulum defined respectively in $R_{f_{1}}$ and $R_{f_{0}}$ can then be calculated as:

$$
\begin{gathered}
\omega_{\mathbf{x}}^{\mathbf{p}_{1}}=\dot{\alpha} \mathbf{i} \\
\omega_{\mathbf{x}}^{\mathbf{p}_{\mathbf{o}}}=\omega_{\mathbf{x}}^{\mathbf{s}}+T_{1 x}^{0} \omega_{\mathbf{x}}^{\mathbf{p}_{1}}=(\dot{\alpha}-\dot{\theta}) \mathbf{i} \\
\omega_{\mathbf{y}}^{\mathbf{p}_{1}}=\dot{\beta} \mathbf{j} \\
\omega_{\mathbf{y}}^{\mathbf{p}_{\mathbf{0}}}=\omega_{\mathbf{y}}^{\mathbf{s}}+T_{1 y}^{0} \omega_{\mathbf{y}}^{\mathbf{p}_{1}}=(\dot{\beta}-\dot{\phi}) \mathbf{j}
\end{gathered}
$$

The angular velocity vector $\omega^{\mathbf{p}}$ defined in $R_{f}$ is identical to $\omega^{\mathbf{p}_{0}}$.

The linear velocity $\mathbf{v}^{\mathbf{p}}$ of the mass center of the pendulum can then be calculated as:

$$
\begin{aligned}
\mathbf{v}_{\mathbf{x}}^{\mathbf{p}}= & \mathbf{v}_{\mathbf{x}}^{\mathbf{s}}+\omega_{\mathbf{x}}^{\mathbf{p}} \times \mathbf{r}_{\mathbf{x}}^{\mathbf{p}} \\
\mathbf{v}_{\mathbf{x}}^{\mathbf{p}}= & {[-R \dot{\theta}+l \cos (\alpha-\theta)(\dot{\alpha}-\dot{\theta})] \mathbf{j} } \\
& +[l \sin (\alpha-\theta)(\dot{\alpha}-\dot{\theta})] \mathbf{k} \\
\mathbf{v}_{\mathbf{y}}^{\mathbf{p}=} & \mathbf{v}_{\mathbf{y}}^{\mathbf{s}}+\omega_{\mathbf{y}}^{\mathbf{p}} \times \mathbf{r}_{\mathbf{y}}^{\mathbf{p}} \\
\mathbf{v}_{\mathbf{y}}^{\mathbf{p}=} & {[R \dot{\phi}-l \cos (\beta-\phi)(\dot{\beta}-\dot{\phi})]] \mathbf{i} } \\
& +[l \sin (\beta-\phi)(\dot{\beta}-\dot{\phi})] \mathbf{k}
\end{aligned}
$$


2) Decoupled Dynamic Equations: The dynamical modeling equations of the rolling sphere are decoupled as mentioned in Section I. In this approach, the dynamic interaction between rotations of the sphere around the transversal and longitudinal axes is neglected. On the other hand, the rolling motion of the sphere is provided by the internal pendulum, which make the sphere rotate around the transversal and longitudinal axes. The rotational motion of the sphere around the vertical $\mathrm{z}$ axis is assumed to be negligible with respect to other actuated rotations around the transversal and longitudinal axes. Part of the dynamics represented by the coordinate $\psi$ disappears and finally two separate set equations of motion can be written for the translations along the perpendicular axes defining the motion plane.

Let $E_{k}$ and $E_{p}$ denote respectively the total kinetic and potential energy of the system. The Lagrangian function is then written as follows:

$$
L=E_{k}-E_{p}
$$

The total kinetic energy is expressed in terms of the masses, inertia, linear velocities, and angular velocities using the following equation:

$$
E_{k}=\frac{1}{2} M^{s}\left\|\mathbf{v}^{\mathbf{s}}\right\|^{2}+\frac{1}{2} I^{s}\left\|\omega^{\mathbf{s}}\right\|^{2}+\frac{1}{2} m^{p}\left\|\mathbf{v}^{\mathbf{p}}\right\|^{2}+\frac{1}{2} I^{p}\left\|\omega^{\mathbf{p}}\right\|^{2}
$$

where,

$$
\begin{aligned}
I^{s} & =\frac{2}{3} M^{s} R^{2} \\
I^{p} & =\frac{1}{12} m^{p} l^{2}+m^{p}\left(\frac{l}{2}\right)^{2}
\end{aligned}
$$

With $M^{s}$ and $m^{p}$ representing the masses of the sphere and pendulum, $I^{s}$ and $I^{p}$ the mass moment of inertia and $v^{s}, \omega^{s}$, $v^{p}$ and $\omega^{p}$ the linear and angular velocities of the sphere and pendulum.

The total potential energy of the system can be expressed with respect to the center of the sphere:

$$
E_{p}=m^{p} g r^{p-z}
$$

with $r^{p-z}$ being the vertical position of the mass center of the pendulum.

By decoupling the system motion, the Lagrangian function $\mathrm{L}$ can be split into $L_{x}$ and $L_{y}$ including only the terms due to rotations about transversal and longitudinal axes, respectively.

$$
\begin{aligned}
L_{x}= & \frac{1}{2} M^{s}(-R \dot{\theta})^{2}+\frac{1}{2} I^{s}(-\dot{\theta})^{2} \\
& +\frac{1}{2} m^{p}\left[(-R \dot{\theta}+l \cos (\alpha-\theta)(\dot{\alpha}-\dot{\theta}))^{2}\right. \\
& \left.+(l \sin (\alpha-\theta)(\dot{\alpha}-\dot{\theta}))^{2}\right] \\
& +\frac{1}{2} I^{p}(\dot{\alpha}-\dot{\theta})^{2}+m^{p} g l \cos (\alpha-\theta)
\end{aligned}
$$

$$
\begin{aligned}
L_{y}= & \frac{1}{2} M^{s}(R \dot{\phi})^{2}+\frac{1}{2} I^{s}(-\dot{\phi})^{2} \\
& +\frac{1}{2} m^{p}\left[(R \dot{\phi}-l \cos (\beta-\phi)(\dot{\beta}-\dot{\phi}))^{2}\right. \\
& \left.+(l \sin (\beta-\phi)(\dot{\beta}-\dot{\phi}))^{2}\right] \\
& +\frac{1}{2} I^{p}(\dot{\beta}-\dot{\phi})^{2}+m^{p} g l \cos (\beta-\phi)
\end{aligned}
$$

For translation along $O-y$, the Euler-Lagrange equations of the system are written as follows:

$$
\frac{d}{d t}\left(\frac{\partial L_{x}}{\partial \dot{q}_{i}}\right)-\frac{\partial L_{x}}{\partial q_{i}}=Q_{i}
$$

where $q_{1}=\theta$ and $q_{2}=\alpha$ are the generalized coordinates. $Q_{i}$ represents the input torque to act the system. In fact, when the pendulum is rotated through an input torque, a reaction torque about the shaft occurs in the opposite direction [8]. $Q_{1}=\tau_{\theta}$ and $Q_{2}=\tau_{\alpha}$ represent the input torque to rotate the pendulum.

$$
\begin{aligned}
& Q_{\theta}=\tau_{x} \\
& Q_{\alpha}=\tau_{x}
\end{aligned}
$$

For translation along $O-x$, the Euler-Lagrange equations are written similarly as follows:

$$
\frac{d}{d t}\left(\frac{\partial L_{y}}{\partial \dot{q}_{i}}\right)-\frac{\partial L_{y}}{\partial q_{i}}=Q_{i}
$$

where $q_{1}=\phi$ and $q_{2}=\beta$ are the generalized coordinates. $Q_{1}=\tau_{\phi}$ and $Q_{2}=\tau_{\beta}$ represent the input torque to rotate the pendulum.

$$
\begin{aligned}
& Q_{\phi}=\tau_{y} \\
& Q_{\beta}=\tau_{y}
\end{aligned}
$$

The decoupled equations given above can be rewritten in a combined state space with the following generalized coordinates vector:

$$
\mathbf{q}=\left(\begin{array}{llll}
\theta & \alpha & \phi & \beta
\end{array}\right)^{T}
$$

Equations of motion can be finally written in matrix form as follows:

$$
\begin{gathered}
M(q(t)) q \ddot{q(t)}+V(q(t), q \dot{(t)})=u(t) \\
{\left[\begin{array}{llll}
M_{11} & M_{12} & M_{13} & M_{14} \\
M_{21} & M_{22} & M_{23} & M_{24} \\
M_{31} & M_{32} & M_{33} & M_{34} \\
M_{41} & M_{42} & M_{43} & M_{44}
\end{array}\right]\left[\begin{array}{l}
\ddot{q}_{1} \\
\ddot{q}_{2} \\
\ddot{q_{3}} \\
\ddot{q}_{4}
\end{array}\right]} \\
+\left[\begin{array}{c}
V_{11} \\
V_{21} \\
V_{31} \\
V_{41}
\end{array}\right]=\left[\begin{array}{c}
\tau_{x} \\
\tau_{x} \\
\tau_{y} \\
\tau_{y}
\end{array}\right]
\end{gathered}
$$

where 


$$
\begin{aligned}
M_{11}= & M_{s} R^{2}+m_{p} R^{2}+m_{p} l^{2}+I_{s}+I_{p} \\
& +2 m_{p} R l \cos \left(q_{2}-q_{1}\right) \\
M_{12}= & -m_{p} l^{2}-I_{p}-m_{p} R l \cos \left(q_{2}-q_{1}\right) \\
M_{13}= & M_{14}=0 \\
M_{21}= & -m_{p} l^{2}-I_{p}-m_{p} R l \cos \left(q_{2}-q_{1}\right) \\
M_{22}= & m_{p} l^{2}+I_{p} \\
M_{23}= & M_{24}=M_{31}=M_{32}=0 \\
M_{33}= & M_{s} R^{2}+m_{p} R^{2}+m_{p} l^{2}+I_{s}+I_{p} \\
& +2 m_{p} R l \cos \left(q_{3}-q_{4}\right) \\
M_{34}= & -m_{p} l^{2}-I_{p}-m_{p} R l \cos \left(q_{4}-q_{3}\right) \\
M_{41}= & M_{42}=0 \\
M_{43}= & -m_{p} l^{2}-I_{p}-m_{p} R l \cos \left(q_{4}-q_{3}\right) \\
M_{44}= & m_{p} l^{2}+I_{p} \\
V_{11}= & m_{p} R l \sin \left(q_{2}-q_{1}\right) \dot{q}_{1}{ }^{2}+m_{p} R l \sin \left(q_{2}-q_{1}\right) \dot{q}_{2}{ }^{2} \\
& -2 m_{p} R l \sin \left(q_{2}-q_{1}\right) q_{1} q_{2}-m_{p} g l \sin \left(q_{2}-q_{1}\right) \\
V_{21}= & +m_{p} g l \sin \left(q_{2}-q_{1}\right) \\
V_{31}= & +m_{p} R l \sin \left(q_{4}-q_{3}\right) \dot{q}_{3}{ }^{2}+m_{p} R l \sin \left(q_{4}-q_{3}\right) \dot{q}_{4}{ }^{2} \\
& -2 m_{p} R l \sin \left(q_{4}-q_{3}\right) q_{3} q_{4}-m_{p} g l \sin \left(q_{4}-q_{3}\right) \\
V_{41}= & +m_{p} g l \sin \left(q_{4}-q_{3}\right)
\end{aligned}
$$

\section{B. Radius of Curvature over Curvilinear Trajectories}

Free body diagrams of the rolling sphere over a curvilinear trajectory are given in Fig. 2 and Fig.3.

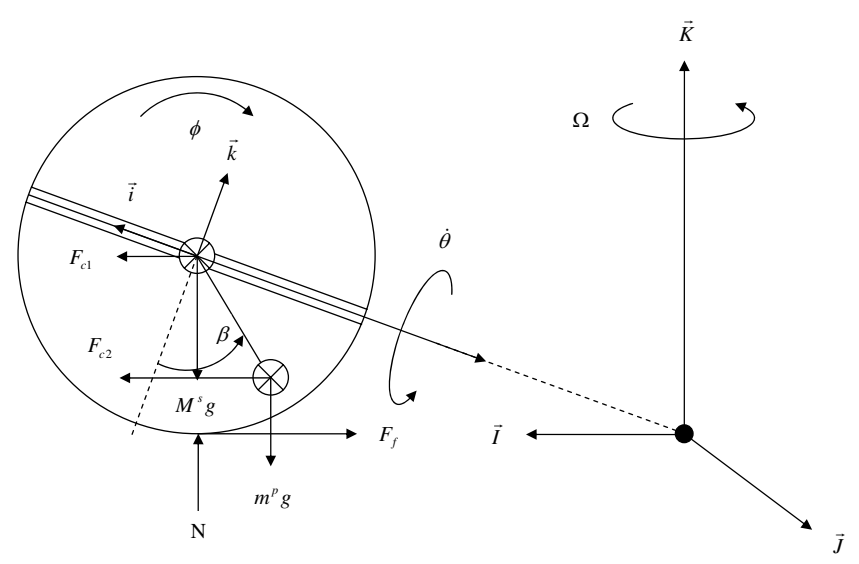

Fig. 3. Forces Acting on the Sphere While Moving Over a Curvilinear Trajectory

The angular velocity of the sphere rotating around a vertical axis (Fig.3) is given by:

$$
\Omega=\frac{-R \dot{\theta}}{e}
$$

where $R, e$ and $\dot{\theta}$ represent the radius of the sphere, the radius of curvature and driving angular velocity of the system, respectively.
Friction force operating at the contact point between the sphere and ground surface compensates for the total centrifugal force acting on the system:

$$
\begin{aligned}
F_{f} & =F_{c 1}+F_{c 2} \\
& =M^{s} e \Omega^{2}+m^{p}(e-l \sin (\beta-\phi)) \Omega^{2} \\
& \approx\left(M^{s}+m^{p}\right) e \Omega^{2}
\end{aligned}
$$

where $F_{c 1}, F_{c}, F_{f}$ and $\phi$ represent the centrifugal force acting on the sphere, centrifugal force acting on the pendulum, the friction force and turning angle of the sphere, respectively.

Torque acting around the transversal axis of the sphere can be expressed as follows:

$$
\begin{aligned}
T_{1}= & m^{p} g l \sin (\beta-\phi)+F_{c 2} l \cos (\beta-\phi)-R F_{f} \\
\approx & m^{p} g l \sin (\beta-\phi)+m^{p} e \Omega^{2} l \cos (\beta-\phi) \\
& -R\left(M^{s}+m^{p}\right) e \Omega^{2}
\end{aligned}
$$

The angular velocity and angular momentum of the sphere are written as follows:

$$
\begin{aligned}
\omega & =\Omega \mathbf{K}-\dot{\phi} \mathbf{J}-\dot{\theta} \mathbf{i} \\
& =\Omega \mathbf{K}-\dot{\phi} \mathbf{J}-\dot{\theta} \cos (\phi) \mathbf{I}-\dot{\theta} \sin (\phi) \mathbf{K} \\
& =-\dot{\theta} \cos (\phi) \mathbf{I}-\dot{\phi} \mathbf{J}+(\Omega-\dot{\theta} \sin (\phi)) \mathbf{K}
\end{aligned}
$$

where $\mathbf{i}, \mathbf{j}, \mathbf{k}$ and $\mathbf{I}, \mathbf{J}, \mathbf{K}$ represent the unit vectors on the coordinate system fixed to the center of the sphere and on the ground, respectively.

$$
\begin{aligned}
\mathbf{L} & =I \omega \\
& =-I^{s} \dot{\theta} \cos (\phi) \mathbf{I}-I^{s} \dot{\phi} \mathbf{J}+I^{s}(\Omega-\dot{\theta} \sin (\phi)) \mathbf{K}
\end{aligned}
$$

The time-derivative of the angular momentum gives the total torque applied to the system:

$$
\begin{aligned}
\mathbf{T}_{\mathbf{2}} & =\frac{d L}{d t}=\mathbf{\Omega} \times \mathbf{L} \\
& =I_{s} \Omega \dot{\phi} \mathbf{I}-I_{s} \Omega \dot{\theta} \cos (\phi) \mathbf{J}
\end{aligned}
$$

Equations (35) and (38) describe respectively the torque around unit vectors $\mathbf{I}$ and $\mathbf{J}$. Torque around unit vectors $\mathbf{I}$ in (38) and (35) are equal to each other. Assume that $\phi$ is small. Radius of curvature is obtained considering (33) and approximately $\phi=0$ as follows:

$$
\begin{aligned}
T_{1}= & T_{2} \\
-I_{s} \Omega \dot{\theta} \cos \phi \approx & m_{p} g l \sin (\beta)+m_{p} e \Omega^{2} l \cos (\beta) \\
& -R\left(M_{s}+m_{p}\right) e \Omega^{2} \\
e \approx & \frac{R(\dot{\theta})^{2}\left[I_{s}-m_{p} R l \cos (\beta)\right]}{m_{p} g l \sin (\beta)} \\
& +\frac{R(\dot{\theta})^{2}\left[R^{2}\left(M_{s}+m_{p}\right)\right]}{m_{p} g l \sin (\beta)}
\end{aligned}
$$


As can be seen from (39), the radius of curvature is related to the angular velocity of the driving motion and the turning angle of the pendulum. Fig. 4 shows this relationship for different angular velocities of driving motion values from 1 to $5 \mathrm{rad} / \mathrm{s}$ for every $1 \mathrm{rad} / \mathrm{s}$ increment. The curves given in Fig. 4 can later serve as references in trajectory generation of the proposed system.

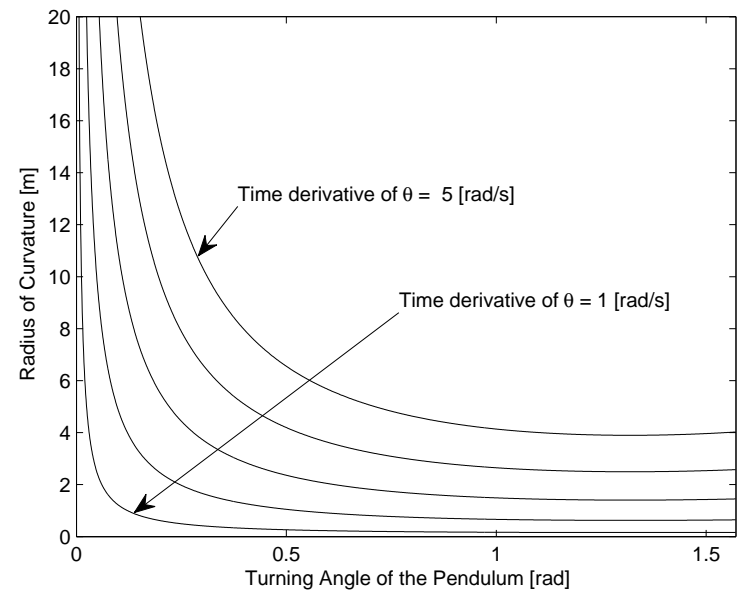

Fig. 4. Radius of Curvature as Function of the Rolling Speed of the Sphere and Turning Angle of the Pendulum

\section{Control of Spherical Rolling Motion}

\section{A. Feedback Linearization}

Feedback linearization is a closed-loop control design for nonlinear systems. The main idea in feedback linearization is to algebraically transform the nonlinear dynamics into linear dynamics through appropriate feedback. The resulting closed-loop linear dynamics is then easily governed by linear control techniques. Feedback linearization is frequently used in control of serial manipulators and known as "computed torque control" in robotics literature.

Equations of motion of mechanical systems are generally formalized as follows:

$$
M(q(t)) q \ddot{(t})+V(q(t), \dot{q(t)})=u(t)
$$

With the so-called linearizing control law $u(t)$ designed as follows:

$$
\begin{aligned}
u(t)= & V\left(q(t), q \dot{q(t))}+K_{v} \dot{e(t)}+K_{p} e(t)\right. \\
& +M(q(t)) \ddot{q_{d}}(t)
\end{aligned}
$$

The resulting closed-loop error dynamics is given by:

$$
e \ddot{(t)}+K_{v} e \dot{(t)}+K_{p} e(t)=0
$$

where $K_{p}$ and $K_{v}$ are positive definite feedback gains.

The equations of motion governing the rolling dynamics have been decoupled as presented in Section III. With feedback linearization, the nonlinearities present in the decoupled equations (31) will be compensated in closed-loop. The block diagram of a feedback linearization scheme with PD controller applied in simulations is shown in Fig. 5.

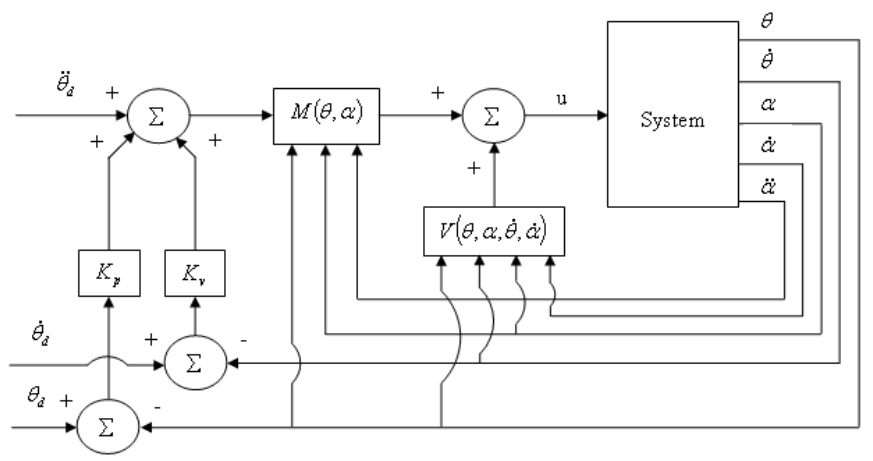

Fig. 5. Block Diagram of a PD Type Controller Structure for Feedback Linearization

\section{B. Fuzzy Control}

In physical applications, structural and parametric uncertainties such as unmodeled dynamics and physical disturbances cause unwanted effects on the system behaviour. Fuzzy control actions can be added to the control law in order to increase the robustness of the system.

A fuzzy controller with two inputs and one output is considered in the control of the system. One of the inputs is the position error and the other is the velocity error, i.e. the rate of change of the position error. With the introduction of the fuzzy controller, the constant PD gains will be transformed into time-varying parameters. The block diagram of the PD type fuzzy controller applied in simulations is shown in Fig. 6.

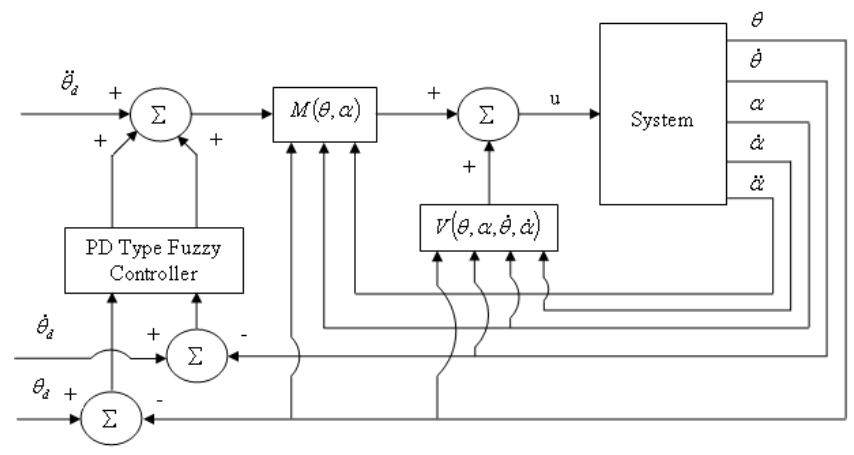

Fig. 6. Block Diagram of a PD Type Fuzzy Controller Structure for Feedback Linearization

The rule base used by the fuzzy controller is shown in Table II. The first column and row are the linguistic results of the error and the rate of change of the error respectively. The membership functions of inputs and outputs are chosen as triangular functions as shown in Fig. 7. 
TABLE II

RUle BASE FOR A PD TYPE FuZZy CONTROLler

\begin{tabular}{|c|c|c|c|c|c|c|c|}
\hline$e l \dot{e}$ & NL & NM & NS & ZR & PS & PM & PL \\
\hline PL & ZR & PS & PM & PL & PL & PL & PL \\
\hline PM & NS & ZR & PS & PM & PL & PL & PL \\
\hline PS & NM & NS & ZR & PS & PM & PL & PL \\
\hline ZR & NL & NM & NS & ZR & PS & PM & PL \\
\hline NS & NL & NL & NM & NS & ZR & PS & PM \\
\hline NM & NL & NL & NL & NM & NS & ZR & PS \\
\hline NL & NL & NL & NL & NL & NM & NS & ZR \\
\hline
\end{tabular}

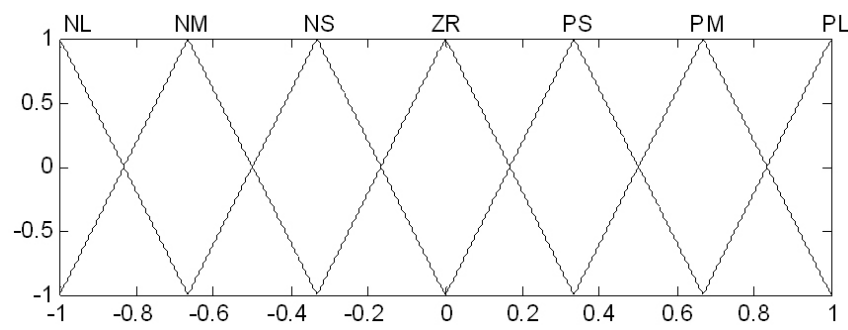

Fig. 7. The membership functions of inputs and output

\section{Simulation Results}

The following numerical values have been used in the simulations: $M^{s}=3 \mathrm{~kg}, m^{p}=2 \mathrm{~kg}, R=0.2 \mathrm{~m}, l=0.075$ $\mathrm{m}$ and $g=9.81 \frac{\mathrm{m}}{\mathrm{s}^{2}}$. The input torque $\mathrm{u}$ is limited by a saturation function with a maximum value of $2.5 \mathrm{Nm}$. The system equations are sampled with a sampling period of 0,001 $\mathrm{s}$ for linear trajectories and $0,001 \mathrm{~s}, 0,1 \mathrm{~s}$ and $0,15 \mathrm{~s}$ for curvilinear trajectories.

\section{A. Rolling Over Linear Trajectories}

Fig. 8 and 9 show examples of step responses of the system for various PD gains. The required input torque for various PD gains are given in Fig. 10. The simulation results show that the system's time response becomes faster with increasing proportional gain $K_{p}$. The maximum overshoot is also proportional to $K_{p}$, as expected. The gains are selected such that the required input torque does not exceed the maximum value allowed by the actuators, i.e. $2.5 \mathrm{Nm}$. Proportional and derivative gains are determined as $K_{p}=1$ and $K_{v}=1$. Required input torque for the selected values of the gains is given in Fig. 11.

Fig. 12 compares the step responses with PD type and PD type fuzzy controllers. The PD type fuzzy controller gives a smaller rise time, overshoot and settling time. Fig. 13 shows the required input torque with PD type fuzzy controller. It can be seen that fuzzy control action reduces the total required torque for the system motion.

As can be seen in below figures, the velocity error of linear trajectories settles down in less than 7 seconds by using PD type fuzzy controllers.

\section{B. Rolling Over Curvilinear Trajectories}

The simulation results for tracking of a circular trajectory with PD type and PD type fuzzy controllers are respectively given in Fig. 14, 15, 16, 17, 18 and 19. The simulation results show that the tracking error can be decreased with the

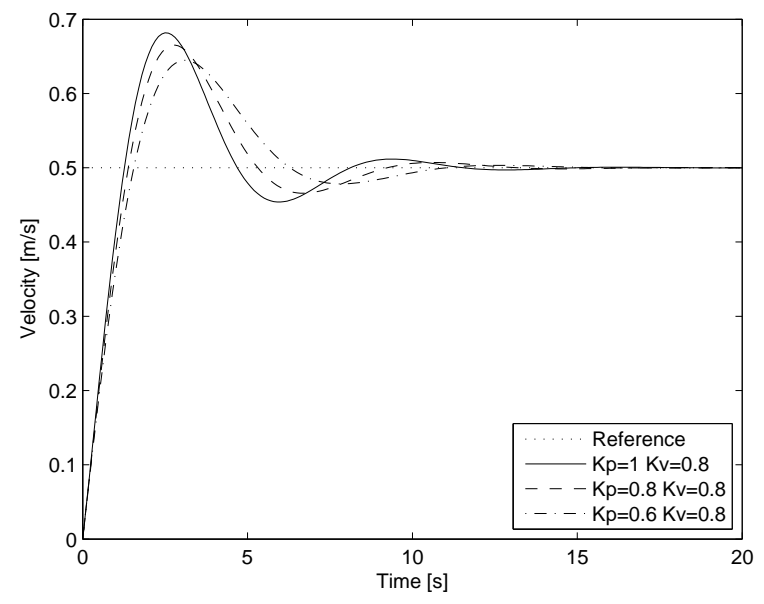

Fig. 8. Step responses for various proportional gains

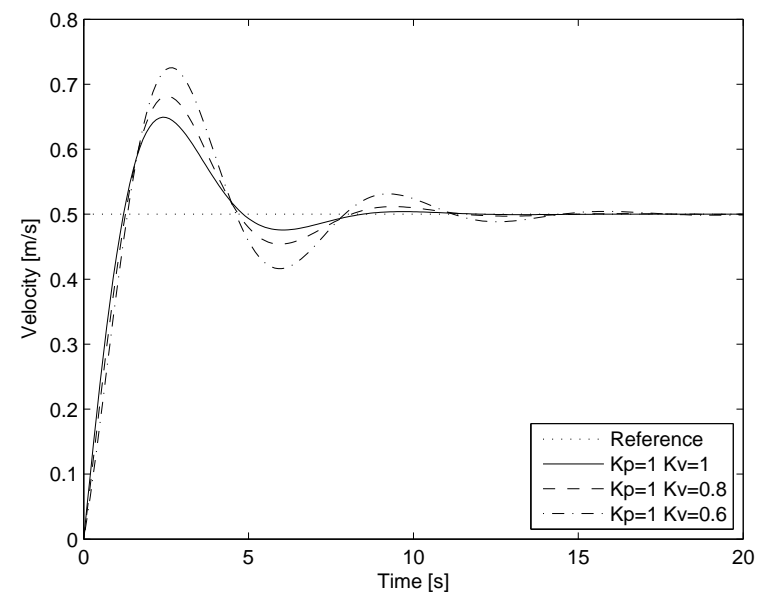

Fig. 9. Step Responses for various derivative gains

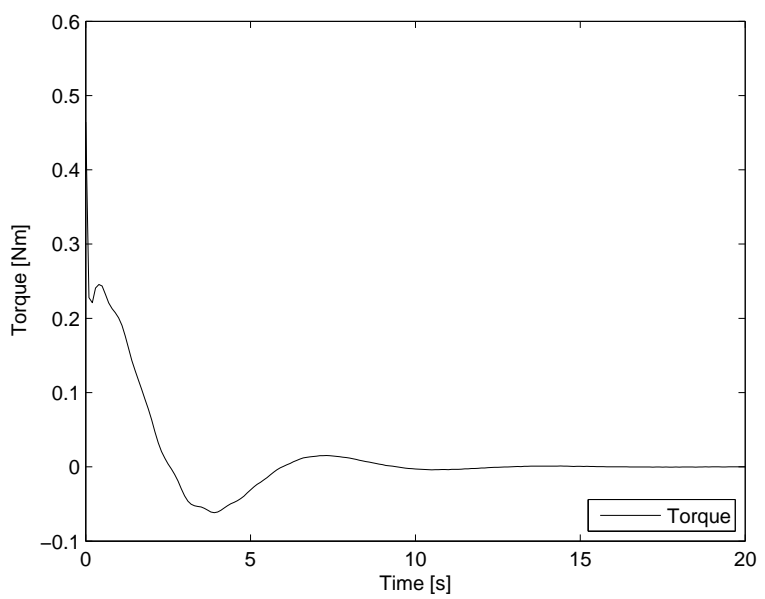

Fig. 10. Required input torque for $\mathrm{Kp}=1$ and $\mathrm{Kv}=0,8$

additional fuzzy control action with respect to the simple PD 


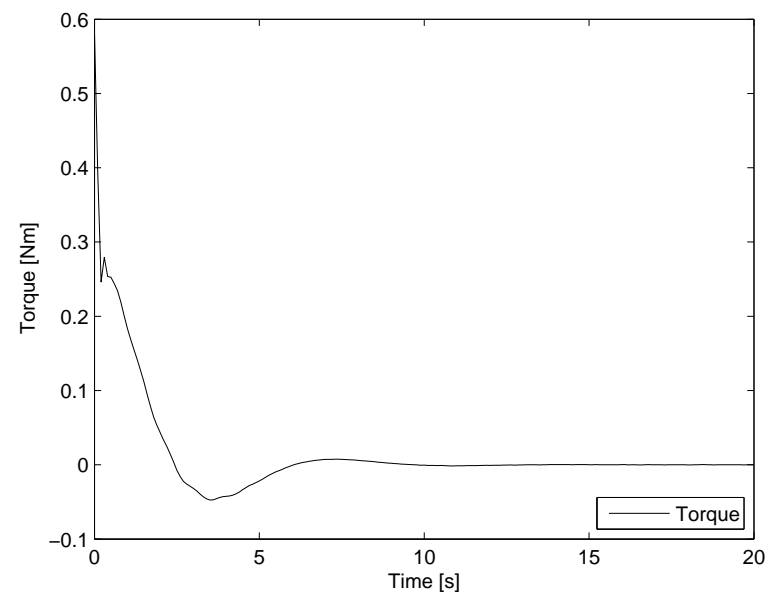

Fig. 11. Required input torque for $\mathrm{Kp}=1$ and $\mathrm{Kv}=1$

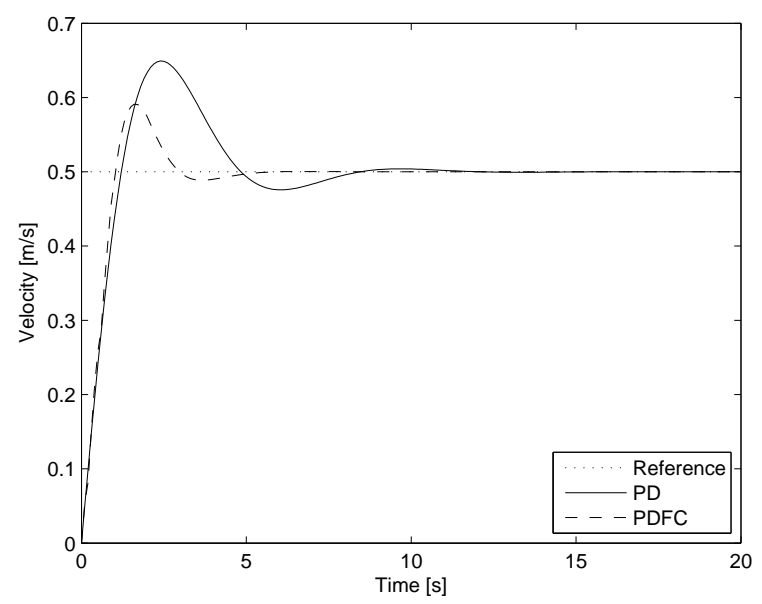

Fig. 12. Step responses with PD and PD Fuzzy Controller (PDFC)

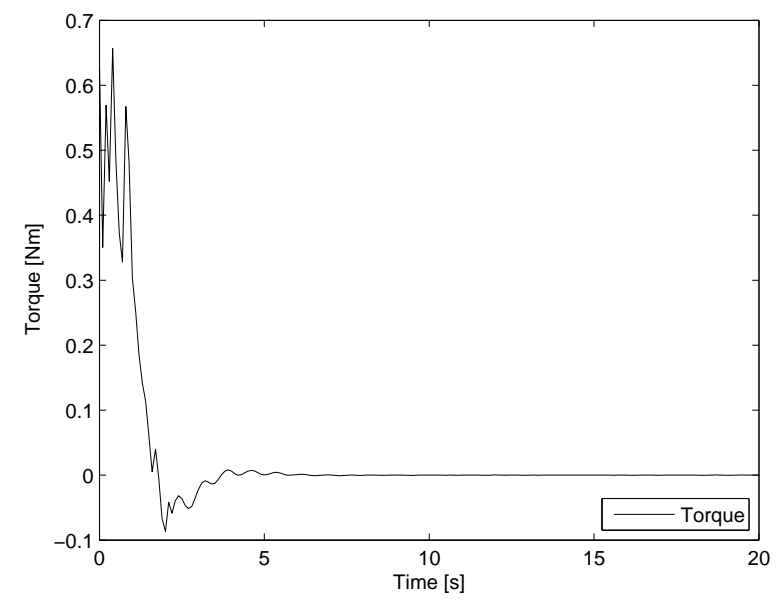

Fig. 13. Required input torque with PD Fuzzy Controller (PDFC)

control. The tracking error increases with increasing values of the sampling period as expected.

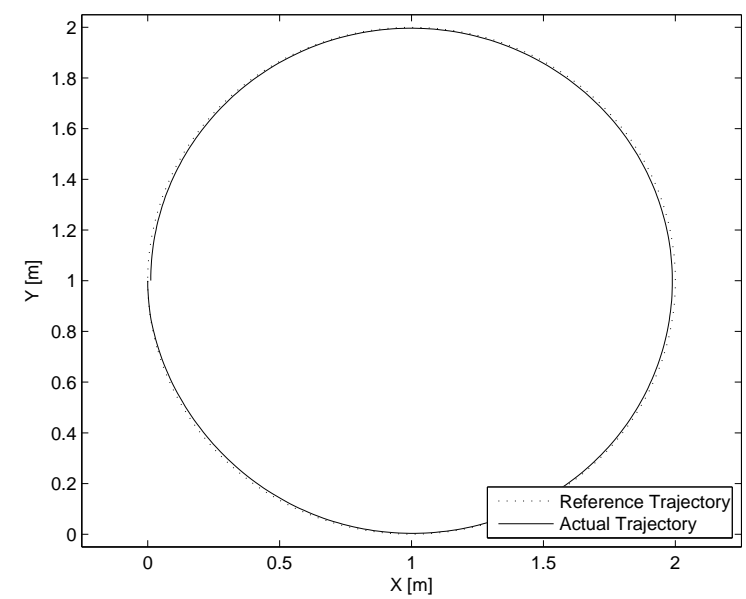

Fig. 14. PD Type Controller - sampling period of $0.001 \mathrm{~s}$

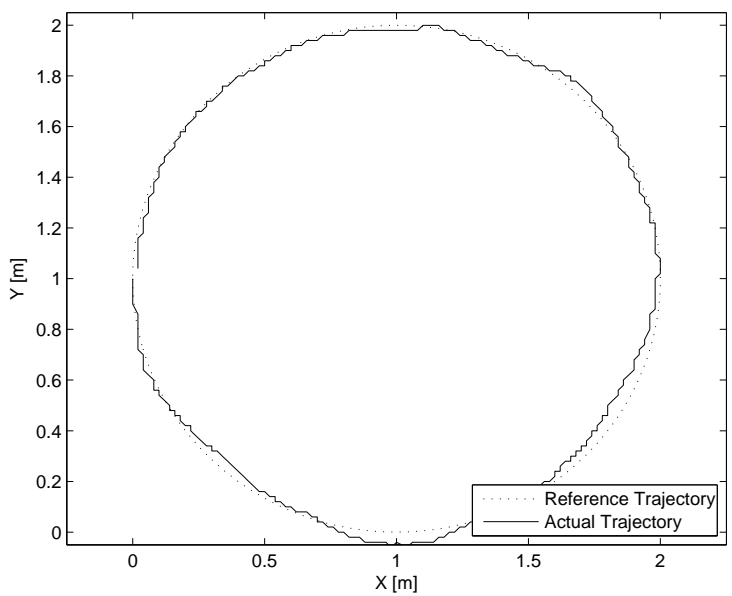

Fig. 15. PD Type Controller - sampling period of $0.1 \mathrm{~s}$

\section{CONCLUSION}

In this study, modeling, analysis and control aspects of a spherical rolling robot have been investigated. The proposed spherical mechanism consists of a pendulum with 2 DOFs which provides the $2 \mathrm{D}$ rolling motion around the transversal and longitudinal axes of the sphere. Highly nonlinear and coupled equations of motion along with nonholonomic constraints have been derived using the Euler-Lagrange method. Rolling motions around the transversal and longitudinal axes have then been decoupled in order to obtain a simpler state-space formulation for the equation of dynamic model. The radius of curvature over curvilinear trajectories, has also been analyzed. Classical feedback linearization and feedback linearization with fuzzy controllers have been designed for closed-loop control of the rolling motion over linear and curvilinear trajectories. The performance of the proposed mechanical model and control schemes have been validated in dynamic simulations. 


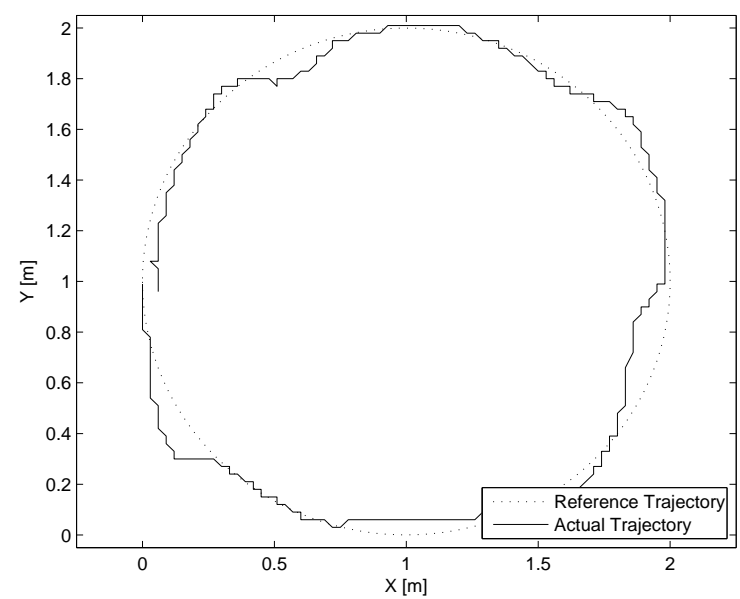

Fig. 16. PD Type Controller - sampling period of $0.15 \mathrm{~s}$

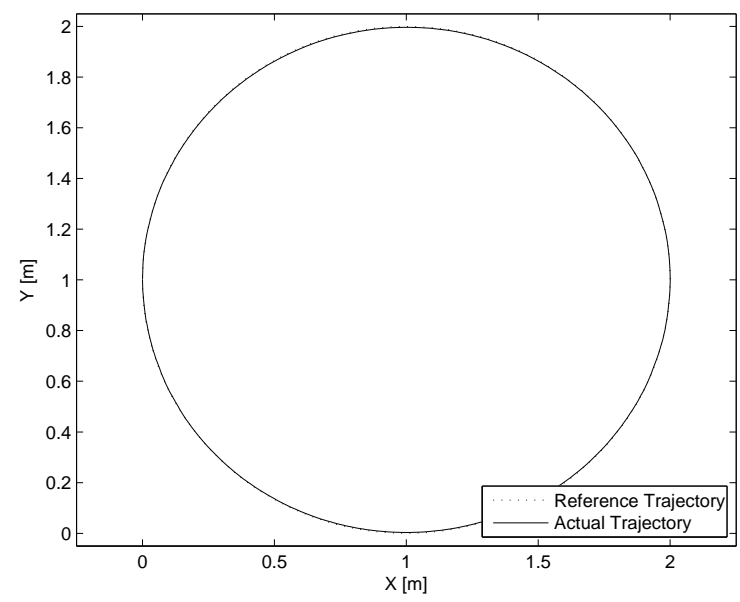

Fig. 17. PD Type Fuzzy Controller - sampling period of $0.001 \mathrm{~s}$

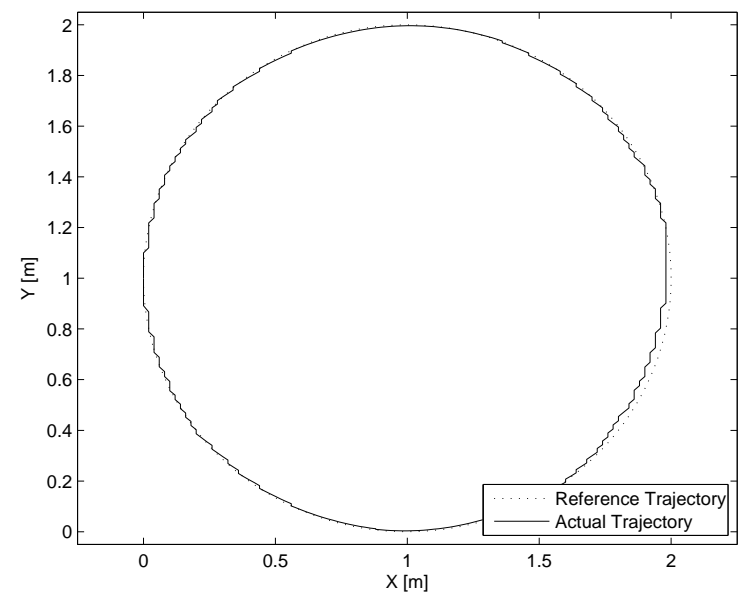

Fig. 18. PD Type Fuzzy Controller - sampling period of $0.1 \mathrm{~s}$

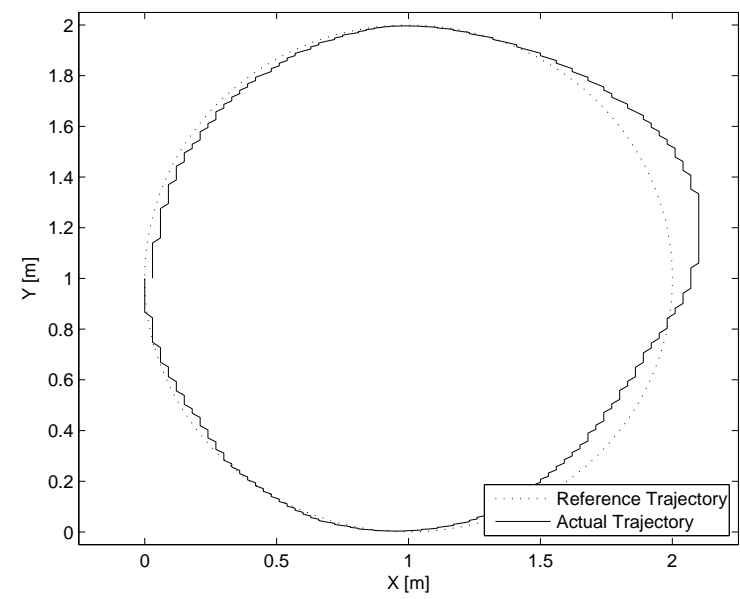

Fig. 19. PD Type Fuzzy Controller - sampling period of $0.15 \mathrm{~s}$

In conclusion, equations of motion of spherical rolling have been simplified by decoupling highly complex original rolling dynamics. We have observed that the proposed fuzzy control action resulted with better closed-loop behaviour with respect to conventional PD control. Since overshoot in step response is about $\% 20$, additional control actions such as grey prediction can be considered for the compensation of unwanted transient behaviour.

\section{REFERENCES}

[1] A. Bicchi, A. Balluchi, D. Prattichizzo, and A. Gorelli, "Introducing the sphericle: an experimental testbedfor research and teaching in nonholonomy," in Proceedings of the IEEE International Conference on Robotics and Automation, Albuquerque, NM, USA, 1997, pp. 2620 2625.

[2] J. Alves and J. Dias, "Design and control of a spherical mobile robot," Proceedings of the I MECH E Part I Journal of Systems and Control Engineering, vol. 217, pp. 457-467, 2003.

[3] C. Camicia, F. Conticelli, and A. Bicchi, "Nonholonomic kinematics and dynamics of the sphericle," in Proceedings of IEEE/RSJ International Conference on Intelligent Robots and Systems, vol. 1, Takamatsu, Japan, 2000, pp. $805-810$.

[4] A. Halme, T. Schonberg, and Y. Wang, "Motion control of a spherical mobile robot," in Proceedings of fourth International Workshop on Advanced Motion Control, vol. 1, Albuquerque, NM, USA, 1996, pp. 259-264.

[5] A. A. H. Javadi and P. Mojabi, "Introducing august: a novel strategy for an omnidirectional spherical rolling robot," in Proceedings of the IEEE International Conference on Robotics and Automation, vol. 4, Washington DC, USA, 2002, pp. 3527-3533.

[6] - , "Introducing glory: A novel strategy for an omnidirectional spherical rolling robot," Journal of Dynamic Systems, Measurement, and Control, vol. 126, pp. 678-683, 2004.

[7] R. Mukherjee, M. A. Minor;, and J. T. Pukrushpan, "Simple motion planning strategies for spherobot: a spherical mobile robot," in Proceedings of the 38th IEEE Conference on Decision and Control, vol. 3, Washington DC, USA, 1999, pp. 2132 - 2137.

[8] Y. Ming, D. Zongquan, Y. Xinyi, and Y. Weizhen, "Introducing hit spherical robot: Dynamic modeling and analysis based on decoupled subsystem," in Proceedings of the IEEE International Conference on Robotics and Biomimetics, Harbin, China, 2006, p. 181186.

[9] V. A. Joshi and R. N. Banavar, "Motion analysis of a spherical mobile robot," Robotica, vol. 27, pp. 343 - 353, 2009.

[10] V. A. Joshi, R. N. Banavar, and R. Hippalgaonkar, "Design and analysis of a spherical mobile robot," Mechanism and Machine Theory, vol. 45, pp. $130-136,2009$. 
[11] S. Bhattacharya and S. K. Agrawal, "Spherical rolling robot: a design and motion planning studies," IEEE Transactions on Robotics and Automation, vol. 16, pp. 835-839, 2000.

[12] — "Design, experiments and motion planning of a spherical rolling robot," in Proceedings of the International Conference on Robotics and Automation, vol. 2, San Francisco, CA, USA, 2000, pp. 1207 - 1212.

[13] A. Rosen, "Modified lagrange method to analyze problems of sliding and rolling," Journal of applied mechanics, vol. 67, pp. 697-704, 2000.

[14] A. M. Bloch, Nonholonomic mechanics and control. New York, USA: Springer, 2004.

[15] Z. Qiang, L. Zengbo, and C. Yao, "A back-stepping based trajectory tracking controller for a non-chained nonholonomic spherical robot," Chinese Journal of Aeronautics, vol. 21, pp. 472-480, 2008.

[16] W. Zhuang, X. Liu, C. Fang, and H. Sun, "Dynamic modeling of a spherical robot with arms by using kane's method," in Proceedings of Fourth International Conference on Natural Computation, vol. 4, Beijing, China, 2008, pp. 373-377.

[17] L. A. Gonzalez, "Design, modelling and control of an autonomous underwater vehicle," Ph.D. dissertation, The University of Western Australia, October 2004.

[18] J. Kennedy, "Decoupled modelling and controller design for the hybrid autonomous underwater vehicle: Maco," Ph.D. dissertation, University of Victoria, 2002.

[19] W. Sienel, "Robust decoupling for active car steering holds for arbitrary dynamic tire characteristics," in Proceedings of 3rd European Control Conference, Rome, Italy, 1995, p. 744748.

[20] J. Ackermann and T. Bunte, "Handling improvement for robustly decoupled car steering dynamics," in Proceedings of the 4th IEEE Mediterranean Symposium on New Directions in Control and Automation, Materne, Krete, Greece, 1996, pp. 83- 88.

[21] R. Nakajima, T. Tsubouchi, S. Yuta, and E. Koyanagi, "A development of a new mechanism of an autonomous unicycle," in Proceedings of 1997 IEEE/RSJ International Conference on Intelligent Robots and Systems, vol. 2, Grenoble, France, 1997, pp. 906-912.

[22] A. Schoonwinkel, "Design and test of a computer stabilized unicycle," $\mathrm{Ph} . \mathrm{D}$. dissertation, University of Stanford, 1987.

[23] R. Miklosovic and Z. Gao, "A dynamic decoupling method for controlling high performance turbofan engines," in Proceedings of the 16th IFAC World Congress, Prague, Czech Republic, 2005.

[24] K. W. Au and Y. Xu, "Decoupled dynamics and stabilization of single wheel robot," in Proceedings of 1999 IEEE/RSJ International Conference on Intelligent Robots and Systems, vol. 1, Kyongju, Korea, 1999 , pp. $197-203$.

[25] N. A. Lemos, "Nonholonomic constraints and voronec's equations," arXiv:physics/0306078v1 [physics.ed-ph], June 2003.

[26] L. N. Hand and J. D. Finch, Analytical Mechanics. Cambridge: Cambridge University Press, 1998. 